Revue de l'Institut des langues et cultures

d'Europe, Amérique, Afrique, Asie et Australie

$41 \mid 2020$

Escrituras nómadas en el mundo hispánico contemporáneo

\title{
Au cœur des migrations : les poétiques nomades de Abilio Estévez
}

In the Heart of Migrations: Abilio Estévez's Nomadic Poetics

\section{Renée Clémentine Lucien}

\section{OpenEdition}

Journals

Édition électronique

URL : http://journals.openedition.org/ilcea/10843

DOI : $10.4000 /$ ilcea. 10843

ISSN : 2101-0609

Éditeur

UGA Éditions/Université Grenoble Alpes

Édition imprimée

ISBN : 978-2-37747-224-6

ISSN : 1639-6073

Référence électronique

Renée Clémentine Lucien, «Au cœur des migrations : les poétiques nomades de Abilio Estévez », ILCEA [En ligne], 41 | 2020, mis en ligne le 03 novembre 2020, consulté le 16 février 2021. URL : http:// journals.openedition.org/ilcea/10843; DOI : https://doi.org/10.4000/ilcea.10843

Ce document a été généré automatiquement le 16 février 2021.

(C) ILCEA 


\title{
Au cœur des migrations : les poétiques nomades de Abilio Estévez
}

\author{
In the Heart of Migrations: Abilio Estévez's Nomadic Poetics
}

Renée Clémentine Lucien

1 Avant même que le Cubano-espagnol Abilio Estévez, né en 1959 dans le quartier havanais de Marianao, auteur de nouvelles, romancier et essayiste, n'émigrât à Barcelone en 2000 puis à Palma de Majorque, le nomadisme hantait déjà son écriture. Son œuvre s'est tissée de résonances diverses consubstantielles à « la pensée nomade » théorisée par Gilles Deleuze et Félix Guattari (Deleuze, Guattari, 1980) qui sous-tend sa poétique rebelle à l'unicité et réfractaire aux confortables commodités de la territorialisation. De fait, elle n'a cessé de se complexifier dans des univers mondialisés au gré d'un parcours intime, géographique, psychique, une expérience fondatrice de ses identités et sans cesse propice aux échanges et à la confrontation des imaginaires littéraires et humains. Écrivain de la diaspora cubaine, il forge «[...] la symbiose des paroles, celles que l'on porte en soi, celle du pays quitté et recomposé dans l'imaginaire et celles des lieux que l'on traverse et où l'on va se fixer » (Diard, 2018), enrichissant de la sorte son hybridité caribéenne originelle. La déterritorialisation par la mise en branle d'un voyage réel ou fictif, soit par le biais du rêve et de l'imaginaire, soit par celui de l'exil de ses personnages, trace une colonne vertébrale de la poétique de nombre de ses romans, Los palacios distantes (2002), El navegante dormido (2008), El bailarín ruso de Montecarlo (2010), Archipiélagos, où les frontières entre les motifs littéraires du voyage et du rêve se brouillent souvent, installant les personnages dans un flou territorial et ubiquitaire. La littérature y construit des mises en abyme où les plaisirs prodigués par la beauté sous toutes ses formes fécondent un imaginaire de l'ailleurs, une poétique du nomadisme dans des représentations d'espaces du désir et de l'amour de la vie.

2 Dans Inventario secreto de La Habana (2004), le premier texte publié après son entrée en exil, les romans El bailarín ruso de Montecarlo (2010), Archipiélagos (2015) et la nouvelle 
Jamaica qui fera l'objet d'une prochaine publication ${ }^{1}$, nous montrerons que cette poétique nourrie dès le départ par un tropisme du mouvement, qui a partie liée avec un processus de déterritorialisation, patent dans le motif du voyage réel ou fictif, continue de creuser son sillon et d'être portée par une tonicité amplifiée par la prégnance de la migration globalisée. Ainsi, l'écriture du nomadisme configure une isotopie, dessine une géographie qui tient, non seulement de l'éphémère, du transitoire et de l'entredeux, mais aussi de l'inévitable confrontation à l'altérité, de la porosité entre frontières transformées en lieux de contact, où se côtoient les phénotypes, se créolisent les langues, s'entrechoquent et s'hybrident les imaginaires, entre l'errance, l'ubiquité, la liminalité, et la Relation selon l'acception d'Édouard Glissant (Glissant, $1997: 22$ ), à l'œuvre dans la fiction narrative de Abilio Estévez.

\section{Déterritorialisation, errance, liminalité et ubiquité}

3 Le processus de déterritorialisation est entamé dès le premier roman, Tuyo es el reino, de 1997, qui obtint le Prix du roman étranger en France, en propulsant des personnages érigés en microcosme de la cubanité dans une dynamique spatio-temporelle où l'ici, l'île de l'enfermement, une Cuba jamais nommée, guettée par un prochain cataclysme, un cyclone ravageur, se tend vers un là-bas libérateur. Le franchissement du mur au sens propre et métaphorique leur ouvre des horizons où ils donnent libre cours à leurs fantasmes et à leurs désirs les plus échevelés, qu'ils soient artistiques, érotiques, prosaïques ou élevés. Le motif obsessionnel de l'évasion se décline dans cet espace amphibologique sous des formes multiples dans une poétique où une intertextualité d'une infinie richesse est utilisée comme une arme de transgression sans limites. Ce tropisme de la déterritorialisation avait déjà sans doute semé ses ferments dans l'imaginaire du nomadisme de l'enfant Abilio, dès l'instant où sa mère lui avait lu des pages des Mille et une nuits, ainsi qu'il l'évoque lui-même dans un texte très intimiste sur son inclination envers la déambulation imaginaire par-delà l'océan et son goût de la rêverie, ¿Por qué escribo?, motif qui réapparaîtra intratextuellement tout au long de son œuvre. Il structure le voyage du migrant cubain Abilio Estévez, instance homodiégétique dans Inventario secreto de La Habana, qui s'inscrit dans la configuration d'un narrateur personnage, d'abord en état de désorientation, en quête de repères, dans une situation de liminalité, laquelle serait, selon le concept d'Arnold Von Gennep, une période où un sujet n'est plus dans son ancien statut mais pas encore dans un nouveau (Von Gennep, 1908-1914), d'ubiquité, d'errance, puis d'ancrage hasardeux dans un nouvel univers, la ville de Barcelone. Car la migration suppose une identité racine, celle de son lieu d'origine, et une identité rhizomatique, dans le sens où Edouard Glissant les théorise (Glissant, 1997 : 21), et le migrant est, qu'il le veuille ou non, happé par le divers et le chaos constitutif de la matière du monde, le Tout-Monde (Glissant, 1997 : 22). Seuls des personnages ayant conscience de leur identité, tel que l'auteur-narrateur, entrent en Relation dans la dynamique de l'errance, dans ce monde où certains se figent dans une opacité glacée tandis que d'autres, à rebours, se confrontent à l'altérité. C'est ainsi que le narrateur est lui-même saisi par la tension qui l'habite, dans un café de Palma de Majorque, et que Glissant traduit en ces termes :

Les corps ou les matières ainsi dévoyées de leur équilibre originel ne sont pas séparées en deux, ou en trois parties, etc. [...], ils changent d'univers tout en perdurant dans l'oblique de leur mouvement, ils rétractent leur nature dans un ailleurs qui ne cesse pas d'être ici (Glissant, $2002: 105)$. 
Toute la poétique déployée dans Inventario secreto de La Habana en rend compte par ce qu'elle met en évidence de la porosité inhérente au nomadisme, tout le contraire d'une conception du monde comme une monade qui figerait de manière systématique la vie des humains, laquelle se développe, à l'inverse, au prix d'une nécessaire traversée des frontières qui nourrit une alchimie d'interactions multiples ad infinitum. La liminalité et l'ubiquité, consubstantielles au nomadisme, déterminent nécessairement l'architecture de la poétique rhizomatique de ce texte hybride où l'on relève d'incessants mouvements d'allées et venues entre des fragments de textes génériquement hétérogènes, soigneusement compilés par l'auteur, des pages de romans, des poèmes, des fragments d'essais consacrés à la ville capitale, dessinant un paysage de La Havane depuis le XVIII ${ }^{\mathrm{e}}$ siècle où Alexandre de Humboldt (Ensayo política de la isla de Cuba, 1800) côtoie Anaïs Nin (Diario, 1922), María Zambrano (Carta a José Lezama Lima, 1953) et Graham Greene (Nuestro hombre en La Habana, 1958), parmi beaucoup d'autres témoins épris de la ville. Ils alternent avec un récit homodiégétique du passé de l'auteurnarrateur parcourant ses recoins secrets, en dévoilant des pans de son identité-racine qui se superposent à des impressions de l'exilé en errance, Abilio Estévez, en déambulation dans les rues de Barcelone ou de Palma. Dès lors, les lieux se télescopent autant que les temporalités. De même, le protagoniste Constantino Augusto de Moreas de El bailarín ruso de Montecarlo vit l'expérience de la déterritorialisation articulée à la liminalité et à l'ubiquité lorsque, venu en Espagne pour prendre part à un colloque sur «José Martí et l'Espagne », il prend la décision de ne pas revenir à Cuba et se retrouve un jour dans une pension de Barcelone, où il éprouve la nette certitude d'être simultanément à La Havane.

He entrado en el cuarto como quien accede a su lugar, a su reino. Dominio nada extraño, al contrario: conocido, conveniente, habitual. - ¿Dónde te he visto antes, cuartucho de Barcelona? - pregunto sonriente, ingenuo como si de pronto hubiera regresado a la adolescencia y como si, en efecto, hubiera estado en aquella habitación alguna vez. (Estévez, $2010: 50)$

Dans la capitale catalane, ce personnage confronte donc sa cubanité à d'autres identités qu'il jauge avec bienveillance, à l'égal de l'auteur-narrateur Abilio Estévez, de Inventario secreto de La Habana. L'attachement immarcescible à la ville-racine dont il ne se départit jamais, d'autant mieux que sa nouvelle condition d'exilé le lui rappelle sans cesse, n'empêche et n'entame en rien la délectation exaltée par son errance dans des villes étrangères où il marche jusqu'à l'épuisement physique, Barcelone, Palma de Majorque, Stuttgart et d'autres villes européennes et américaines devenues archipels du ToutMonde. Il est remarquable qu'en épigraphe de ce texte figurent des citations du Quattor d'Alexandrie de Lawrence Durrell, cet écrivain britannique dont l'œuvre est toute imprégnée de l'appétit envers l'atmosphère urbaine: « $Y$, en cierto sentido, habíamos esperado que la ciudad nos reclamara después de cada ausencia » (Estévez, 2004 : 10). Par exemple, à travers l'errance dans les rues et recoins de Barcelone où sévit une certaine forme de violence, Constantino Augusto de Moreas de El bailarín ruso de Montecarlo ne cherche pas à se soustraire à l'agressivité parfois crapuleuse de délinquants qui lui dérobent son argent car il quête, au contraire, dans la confrontation avec ce qu'Édouard Glissant dénomme l'opacité du divers dans la totalité-monde (Glissant, 1997), des lieux d'échange avec l'Autre, les bars, les territoires où se côtoient les altérités et où l'errance aboutit à une sorte d'utopie du "vivre ensemble", indissociable de l'hybridation des cultures.

ILCEA, 41 | 2020 
L'opacité du divers nous réassemble dans l'absolue diversité, en un tourbillon de rencontres. Utopie qui jamais ne se fixe et qui ouvre demain, comme un soleil et un fruit partagés. (Glissant, 1997 : 27)

6 Dans la nouvelle Jamaica, ce n'est pas le rêve qui produit le sentiment de concomitance temporelle et spatiale mais le souvenir qui s'impose, selon le schéma proustien, de façon très étrange, au personnage cubain Cisco en proie à l'étonnement, d'un autre voyage en compagnie de son père, entre La Havane et un village proche de Santiago de Cuba, près de El Brujo où avait prospéré une plantation de café française au XIX siècle. Ce sentiment advient sous l'effet de sensations éveillées par des images du paysage alors que le train où il voyage se dirige vers le nord de l'Italie. En somme, tout devient phénoménologiquement lieu et objet de contact, comme le théorise James Clifford (Clifford, 1997), au gré de la porosité entre cultures forgée à l'occasion de mouvements, de déplacements, de migrations tout autant géographiques qu'imaginaires, grand motif des poétiques nomades de Abilio Estévez. Ces migrations récusant le fixisme deviennent le foyer de dynamiques identitaires imprévisibles et indéfinissables qui échappent aux catégories déjà répertoriées car fondées sur un incessant partage des humains en errance. C'est pourquoi ces poétiques dessinent une cartographie de l'éphémère et du transitoire, de la reconfiguration de schèmes mentaux, résultant d'une interrogation sur Soi-même et sur l'Autre. C'est à cette condition que s'opère le passage de la frontière, le dépassement du limen par des personnages, et au bout du parcours, que peuvent s'interpénétrer des mondes divers en état de s'écouter et d'échanger.

\section{Géographie et isotopie du nomadisme}

7 La géographie du nomadisme s'articule souvent au motif des regards échangés ou se détournant de l'Autre dans les espaces urbains parcourus par les personnages, dans une poétique éminemment scopique. Le regardeur migrant accoutumé au goût immodéré des Cubains envers le voyeurisme et à leur curiosité sans frein à l'égard des étrangers se trouve d'abord déconcerté et blessé par l'indifférence ou la raideur qu'il perçoit comme une mise en scène spectaculaire des Barcelonais croisés dans la rue quotidiennement, fuyant le regard de l'étranger. En revanche, lorsque l'Autre de l'étranger se fait accueillant, le regard des personnages errants dans l'espace urbain créé par Abilio Estévez caresse avec une jouissance non déguisée ce qui vient d'ailleurs, comme une offrande.

¿Y qué sucede? Que ya no es inocente esto de andar por Barcelona. Ahora indago. Ahora exploro. Registro cada rincón, cada gesto, cada cara. En este instante Barcelona ha dejado de ser una simple ciudad para transformarse en el lugar donde hallar por decirlo solemne $y$ rápidamente "la simultaneidad del tiempo". Se entiende: la concurrencia de "mis" tiempos y la batalla de los hombres (Estévez, 2010 : 122).

C'est aussi cette appréhension du monde environnant qui se dégage de sa rencontre avec Palma de Majorque, ville attrayante pour les oiseaux de passage, comme le dirait l'anthropologue cubain Fernando Ortiz, avec des personnages dont le narrateur peine d'abord à deviner l'origine et qu'il observe intensément, tel ce serveur de café apparemment maghrébin, qu'il imagine être arrivé en patera de l'autre côté de la Méditerranée, plaisantant avec une de ses collègues blonde européenne, probablement allemande, ainsi que se l'imagine l'auteur-narrateur. C'est le lieu d'une liminalité transcendée, où se livre à lui l'humanité de l'Autre, au cœur même de son errance. 
Hay siempre algo humano, entrañable, en el acto de entrar en un café y quitarse la bufanda y la gabardina. Accedes a un sitio público, te despojas del abrigo y experimentas una rara sensación de entrega (Estévez, $2004: 21$ ).

9 Il n'est pas rare que des personnages qui peuplent les fictions de Abilio Estévez voyagent sans papiers et sans bagages, ingrédients d'une isotopie du nomadisme, tel Constantino Augusto de Moreas, ne souhaitant plus se rendre à Saragosse pour participer un colloque consacré au poète national cubain et apôtre de l'indépendance de l'île. Circonspect à l'égard de la représentation de Martí par l'officialité de son pays et quelque peu réticent quant au thème de cette manifestation, il se défait de ses bagages et de ses papiers d'identité, une fois débarqué à Barcelone, et en dépit de sa boiterie, se sent immédiatement plus léger et en proie à une incoercible félicité : «Señor cojo, feo sin exageración, anda con rapidez gracias a las pocas cosas que acarrea " (Estévez, 2010 : 23). Tout aussi symboliquement, le protagoniste Cisco de la nouvelle Jamaica, archétypal des migrants du monde globalisé du début du xxI ${ }^{e}$ siècle, et la jeune femme Kyra accomplissant enfin son rêve de se rendre à Paris, partagent avec Constantino cette aptitude à s'émanciper de liens trop contraignants qui fixent les êtres dans une identité définitive et univoque, et qui opèrent comme un frein à leur écoute de l'altérité. Pris d'une ivresse de la déambulation, Cisco a tant marché dans Milan qu'il n'a pas dormi pendant plus de vingt heures, et avant de monter dans le train en direction de Zurich, s'aperçoit qu'il a oublié ses bagages. Et lorsque s'engage la conversation entre lui et les deux autres migrants du compartiment, après que le jeune Sénégalais Nadir et la Bosniaque musulmane eurent décliné leur identité, précisé leur destination et expliqué les raisons de leur voyage, Cisco hésite à révéler son origine cubaine et commence par emprunter des chemins de traverse avant de s'affirmer comme tel, méfiant à l'endroit d'une certaine représentation et d'un imaginaire stéréotypés attachés à Cuba :

Nunca he estado en Bosnia ni en Senegal, nunca he estado en el este de Europa ni en África, admitió compungido. No crea que haya nada de bosnio en mi país, sí mucho de africano, indudable, mi país es algo africano, ¿sabe? ¿Es usted español?, preguntó Kira. Un poco, no y sí, un poco español y un poco africano, de ultramar [...] Soy cubano, admitió con reticencia. Suspiró. Siempre que reconocía su nacionalidad, terminaba suspirando. ¿Cubano de Cuba?, preguntó Kira. Estuvo a punto de responder No, cubano de Malasia. Se contuvo, le pareció un chiste malo y grosero [...] Fidel Castro, dijo Kira. Che Guevara, dijo Nadir. Él agregó José Lezama Lima, Wifredo Lam, Ernesto Lecuona, Bola de Nieve. Volvió a suspirar con mayor énfasis [...] (Estévez, Jamaica).

Corrélativement, il est remarquable que le motif du passage de la frontière participe de l'isotopie de la migration et du nomadisme qui charpente une bonne partie de l'œuvre car, tout bien considéré, ce motif dessine une typologie représentative des sujets en mouvement dans le monde globalisé. Ces personnages obéissent à des impératifs multidimensionnels. Ceux-ci peuvent leur avoir été assénés par le déchaînement des forces naturelles, tel Teo Martinica, du roman Archipiélagos, transfuge de la capitale Saint Pierre, de son île de la Martinique, dévastée par l'éruption de la Montagne Pelée du 8 mai 1902, et parcourant l'archipel caribéen dont il prend conscience de la diversité insoupçonnée, et qui s'évanouit de Marianao où il avait échoué, sans crier gare, en poursuivant son chemin vers des territoires ignorés après qu'il eut construit une caravelle. Cette caravelle fabriquée avec des débris de planches rejetés par la mer sur les rives cubaines prend tout son sens en tant que mise en abyme de ce que sont les migrations dans l'un des premiers espaces de globalisation de l'ère moderne, la Caraïbe (Estévez, 2015 : 406). Dès lors, Marianao, quartier de naissance de l'écrivain cubain, est 
érigé en théâtre d'une fiction qui érige ce lieu en terre d'accueil d'un processus migratoire intercaribéen. Quant à Constantino, après son séjour dans la pension de Barcelone, il s'émancipe de la pesanteur d'un passé d'enfermement et chemine vers la France, semblable aux passagers du train de Jamaica en route vers des destinations européennes qui seront peut-être transitoires, ignorant s'ils reviendront dans leur terre d'origine. Ainsi en va-t-il de Nadir le Sénégalais, espérant retrouver ses parents en Afrique après avoir rendu visite à ses frères à Clermont-Ferrand, et Kira la Bosniaque fascinée par Paris. Le train qui se meut de Milan vers la Suisse et transporte Cisco venu d'abord à Séville pour des recherches aux Archives des Indes, puis installé à Sienne depuis deux mois, devient le lieu d'une réflexion sur la nature des frontières, sur leur nécessité historique et politique, sur ce qu'elles expriment de la vie des hommes, et d'une comparaison entre le monde connu par le personnage et celui qu'il explore. La frontière terrestre entre l'Italie et la Suisse, si aisément franchie, l'invite à s'interroger sur la spécificité des frontières insulaires. La métaphore suggestive de la cicatrice relative à la frontière des îles ne manque pas d'attirer l'attention par le lien qu'elle établit avec l'exil, source d'une douleur irréparable causée aux hommes, jouets involontaires de tragédies de l'Histoire dont il se sent solidaire car les pensées de Cisco sont empreintes d'une émotion vibrante dans l'anaphore traduisant l'acuité de la blessure :

Hasta esa mañana, pensó, nunca había hecho un viaje en tren que implicara dos países. Nunca entendió bien el concepto de frontera y tampoco es que ahora lo entendiera mucho. Siempre había imaginado una muralla interminable, custodiada por banderas y puestos militares, una marca dolorosa en la tierra, una grieta. Y si las fronteras son las cicatrices de la historia, ¿qué sucede con los países que carecen de fronteras? Si la frontera es el mar, ¿cuál es la herida?, ¿cuál es la historia, cuáles las cicatrices de las islas? (Estévez, Jamaica)

De cette errance à travers une géographie de l'éphémère, en l'occurrence celle que dessine le voyage en train, le passage par cette zone de contact, au sens où l'entend James Clifford (Clifford, 1997) dans son essai Routes and Travels, il ressort que ce qui caractérise les personnages, c'est tout autant leur différence d'origine assumée que leur imparable imprégnation par des marques d'une hybridation globalisée.

\section{Langues et échanges}

12 À cet égard, dans la poétique du nomadisme de Abilio Estévez, une part belle est faite à la diversité des langues et aux stratégies d'approche interrelationnelle entre locuteurs hétérogènes dont s'emparent ces oiseaux migrateurs ou étonnants voyageurs. Les propos hésitants échangés par les locuteurs peuvent apparaître sous la forme d'un maillage du texte en italiques par la langue étrangère, soulignant ainsi leur inscription dans un territoire malgré leur caractère marqué, au sens linguistique. Ainsi donc, les processus d'hybridation sont dépeints comme une émanation de la créolisation inséparable de la Relation, se traduisant par le maniement de parlers "composites » (Glissant, 2010: 13). Dans le cas de Teo Martinica dans Archpiélagos, selon le commentaire rapporté du narrateur, surpris par l'étrangeté de ses réalisations discursives, ses interlocuteurs cubains du quartier havanais de Marianao ne peuvent réprimer un sourire en écoutant ses propos remarquables par ce qui s'apparente à de la préciosité et travaillés par une inégalité de registres que ne reconnaît pas la norme. C'est un phénomène courant chez des locuteurs apprenant une langue dans le contexte particulier du nomadisme et peu sensibles à la spécificité de situations d'énonciation. 
Sa pratique d'un parler composite bâti à partir d'un castillan du Siècle d'Or appris à Porto Rico, à l'occasion de sa rencontre avec un constructeur de bateaux noir, surprenant grand lecteur du Quichotte de Miguel de Cervantès (Estévez, $2015: 186)$ et d'un français créolisé de la Martinique (Estévez, 2015 : 181), pouvait paraître étrange et divertissant. Teo chantait aussi dans une langue "absurde», et cependant, il était compris par ses interlocuteurs bien que son parler n'arborât pas, aux dires du narrateur, les signes distinctifs de la brutalité articulatoire des Havanais. Son usage d'une langue castillane tout à la fois approximative et grevée de tournures taxées d'archaïsantes par ses interlocuteurs cubains se parait ainsi des éclats du divers de la nomadisation. Dans cette perspective d'une poétique du nomadisme, viennent à propos ces considérations d'Édouard Glissant :

Je crois qu'il y a une solidarité dans toutes les langues du monde et que ce qui fait la beauté du chaos-monde [...], c'est cette rencontre, ces éclats, ces éclatements dont nous n'avons pas encore réussi à saisir l'économie ni les principes. (Glissant, 1991-2009: 15)

13 Somme toute, Teo Martinica parlait la langue du Tout-Monde en Relation. Dans Jamaica, c'est une langue qui se diffracte et se créolise en entrant en relation avec d'autres parlers. Dans la poétique du nomadisme d'Abilio Estévez plongé dans le multilinguisme du monde globalisé, l'écriture de l'exil, «espace interstitiel », se déploie "entre les codes, les traditions, les langages et les langues ». (Keller-Privat, 2012). Dans le wagon $\mathrm{du}$ train en route vers Zurich, Cisco le Cubain et ses compagnons de voyage échangent des propos dans un mélange où s'entrechoquent les langues globalisées, l'italien, l'anglais, le français (en italiques dans le texte), non pas simplement dans un but pragmatique, pour une communication d'ordre utilitaire, mais pour faire éclater les bornes culturelles institutionnalisées par les frontières et réduire les barrières de l'altérité puisque chacun tente de se faire entendre aussi par une gestuelle et un échange délectable et chaleureux de vivres, dans une sorte d'utopie du vivre ensemble, que reconnaîtrait Édouard Glissant : «Elle nous réassemble dans l'absolue diversité, en un tourbillon de rencontres. Utopie qui jamais ne se fixe et qui ouvre demain, comme un soleil et un fruit partagés. » (Glissant, 2005 : 27) Ainsi la fiction de l'échange dans sa matérialité s'affiche-t-elle comme un kaléidoscope linguistique du monde globalisé, comme un Tout-Monde en mouvement habité par ces migrants réunis dans un train. Le monde globalisé peut-il se construire autrement que dans « la pagaille polyglotte » qui caractérise L'empreinte de l'Ange, de la romancière franco-canadienne Nancy Houston, et dans laquelle sont baignés les personnages de Abilio Estévez ?

Les poétiques nomades de Abilio Estévez traversent des lieux-racine et des territoires archipéliques en embrassant tout autant l'histoire collective faite de migrations depuis les temps qui virent naître La Havane, dans un sens plurivoque, que les trajectoires individuelles d'hommes de notre époque pris dans des mouvements et des brassages incessants et accélérés. Ainsi, la polyphonie de personnages de la fiction narrative, en interrelation, est-elle concomitante de l'homodiégétisme de personnages engagés dans des processus de mutation imparable. Dans la chronotopie du nomadisme de cet écrivain, la géographie des rencontres, de plus en plus étendue, déjoue tout fixisme et, au contraire, joue de l'éphémère et de l'imprévisible. Si les moments de stase volontaires sont ceux consacrés par des personnages au déchiffrement du mystère de l'Autre malgré sa zone d'opacité, l'épigraphe choisie pour El bailarín ruso de Montecarlo et extraite d'un poème d'Anna Ajmatova : "Y parece el camino tan fácil, / blanco en el bosque esmeralda, / adónde conduce, no lo revelaré.../ " est marquée du sceau du mouvement 
permanent, de la complexité de son expression, du mystère de la destination et de l'imprédictible, signes distinctifs du nomadisme.

\section{BIBLIOGRAPHIE}

ALEXANDRE-GARNIER Corinne \& KELLER-PRIVAT Isabelle (2012), Migrations, exils, errances et écritures, Nanterre : Presses Universitaires de Nanterre, <https://books.openedition.org/pupo/2105>. CLIFFORD James (1997), Routes. Travel and Translation in the Late Twentieth Century, Cambridge, Massachusetts : Harvard University Press.

CHAMOISEAU Patrick \& Glissant Édouard (2007), Quand les murs tombent : l'identité nationale hors-laloi ?, Paris : Éditions Galaade, Institut du Tout Monde.

DIARD Dominique (2018), « Entre l'ici et l'ailleurs. Louis-Philippe Dalembert l'aède vagabond », S. Bouffartigue, D. Diard \& R. C. Lucien (dir.), Entre Haïti et ailleurs. Louis-Philippe Dalembert. Entre Haïti et ailleurs : Loxias-Colloques 9, <http://revel.unice.fr/symposia/actel/index.html?id=886>. DeleuZe Gilles \& GuATtARI Félix (1980), Mille Plateaux, Paris : Éditions de Minuit. ESTEVEZ Abilio (1997), Tuyo es el reino, Barcelona : Tusquets Editores. ESTEVEZ Abilio (2004), Inventario secreto de La Habana, Barcelona : Tusquets Editores. ESTEVEZ Abilio (2010), El bailarín ruso de Montecarlo, Barcelona : Tusquets Editores. Estevez Abilio (2015), Archipiélagos, Barcelona : Tusquets Editores. ESTEVEZ Abilio (2020), Jamaica (sous presse), Barcelona : Tusquets Editores. GLISSANT Édouard (1997), Traité du Tout-Monde, Poétique IV, Paris : nrf Gallimard. GLISSANT Édouard (2005), La cohée du Lamentin, Poétique V, Paris : nrf Gallimard. GLISSANT Édouard (2009), Philosophie de la Relation, Poésie en étendue, Paris : nrf Gallimard. GLISSANT Édouard (2010), L'imaginaire des langues (entretiens avec Lise Gauvin, 1991-2009), Paris : Gallimard.

VON GENNEP Arnold (1908-1914), Religions, mœurs et légendes : essais d'ethnographie et de linguistique (5 séries), Paris : Mercure de France.

\section{NOTES}

1. Cette nouvelle inédite, qui m'a été communiquée par son auteur, sera prochainement publiée dans un recueil par les Éditions Tusquets de Barcelone. 


\section{RÉSUMÉS}

La poétique du nomadisme de l'écrivain cubano-espagnol Abilio Estévez, sous-tendue par la pensée nomade, dessine une géographie de l'exil, de l'éphémère, du transitoire et de l'entredeux, configure une isotopie du mouvement par le rêve et l'imaginaire, le voyage réel ou fictif. Elle se nourrit de l'inévitable confrontation à l'altérité par la porosité entre frontières transformées en lieux de contact. Dans ces espaces, se côtoient les phénotypes, s'hybrident les identités et les imaginaires et se créolisent les langues.

Cuban-Spanish author Abilio Estévez's nomadic poetics weaves a geography of exile, of the ephemeral, the transitory and the in-between, configures an isotopic movement through dreams and the imaginary, through journey be it real or fictitious. It feeds from the unavoidable confrontation to otherness by way of the porosity of frontiers transformed into places of contact. In these spaces, phenotypes co-exist, identities and imagination forms get hybridised, and languages get creolized.

INDEX

Keywords : Nomadism, exile, identity, otherness, "Relation", multilingualism

Mots-clés : Nomadisme, exil, identité, altérité, Relation, multilinguisme

\section{AUTEUR}

\section{RENÉE CLÉMENTINE LUCIEN}

Sorbonne Université, CRIMIC EA 2561 